\title{
Retos y propuestas ante la inminente implantación de la evaluación de destrezas orales en el examen de lengua extranjera de la futura P.A.U.
}

\author{
Antonio MARTÍNEZ SÁEZ \\ CAMILLE, Universidad Politécnica de Valencia \\ anmarsae@upvnet.upv.es \\ Ana Mercedes SEVILLA PAVÓN \\ CAMILLE, Universidad Politécnica de Valencia \\ ansepa@doctor.upv.es \\ Jesús GARCÍA LABORDA \\ Universidad de Alcalá de Henares \\ jesus.garcialaborda@uah.es \\ Emilia ENRÍQUEZ CARRASCO \\ Departamento de Lingüística Aplicada \\ Universidad Politécnica de Valencia \\ evenriq@idm.upv.es
}

Recibido: septiembre 2010

Aceptado: marzo 2011

\section{RESUMEN}

El Ministerio de Educación ha publicado recientemente un nuevo Real Decreto que regula la Prueba de Acceso a la Universidad y contempla la incorporación de cambios en el examen de lengua extranjera, cuyo objetivo es valorar la comprensión oral y lectora, además de la expresión oral y escrita. Debido a que el hecho de examinar la comprensión y expresión oral del estudiante es un componente nuevo en este examen, los docentes de la enseñanza secundaria han mostrado sus dudas e inquietudes y han empezado a plantearse cómo tendrán que adaptar su metodología docente. Varios proyectos y estudios en la Comunidad Valenciana demuestran que es posible introducir la evaluación de las destrezas orales en la Prueba de Acceso a la Universidad. Este trabajo también presenta una serie de posibilidades para la puesta en práctica real de la evaluación de tales destrezas, considerada especialmente factible mediante sistemas informáticos. La comunicación concluye que los puntos favorables exceden a los negativos, y que los profesores cambian su forma de pensar y muestran actitudes positivas tras experimentar con las interfaces de exámenes orales asistidos por ordenador.

Palabras clave: actitudes, PAU, evaluación, destrezas orales, metodología. 


\section{Défis et suggestions pour l'évaluation des compétences orales dans l'examen de langue étrangère du futur examen d'entrée à l'université en Espagne}

\section{RÉSUMÉ}

Le Ministère de l'Éducation espagnol a récemment publié un nouvel arrêté royal qui régit l'examen d'entrée à l'université et intègre les changements dans le test de langue étrangère, qui vise à évaluer l'écoute et la lecture en plus de l'expression orale et écrite. En raison du fait que la compréhension orale et l'expression de l'étudiant est un nouvel élément dans cet examen, les enseignants des écoles secondaires ont des doutes et des inquiétudes et ont commencé à réfléchir à la façon dont ils vont devoir adapter leurs méthodes d'enseignement. Plusieurs projets et études dans la Communauté de Valence montrent qu'il est possible d'introduire l'évaluation orale dans l'examen d'entrée à l'université. Ce document présente également un certain nombre de possibilités pour la mise en pratique réelle de l'évaluation de ces compétences, notamment considérée comme possible par des systèmes informatiques. La communication conclut l'idée que les points positifs prennent le dessus sur les points négatifs et que les enseignants changent leur façon de penser et montrent des attitudes positives après avoir expérimenté des interfaces d'examens oraux assistés par ordinateur.

Mots-clés : attitudes, PAU, évaluation, compétences orales, méthodologie.

Challenges and Proposals for new Spanish universities pre-entry exam: evaluating oral skills in foreign languages

\section{ABSTRACT}

The Spanish Ministry of Education has recently passed the new legislation that regulates the university entry exam and the introduction of a foreign language test which would include the evaluation of oral and written comprehension and production skills. The evaluation of oral skills is a new component in the foreign language university entry exam. Therefore, the education professionals are expressing their doubts and worries and are already thinking about the way in which these changes would affect or change their current educational methodology. Several projects and studies developed in the Region of Valencia confirm that the introduction of the evaluation of oral skills in the university entry examination (PAU) is possible. This paper presents a series of possibilities which would permit the evaluation of these skills, which is especially feasible through computer assisted systems. This article concludes by mentioning that the positive aspects exceed the negative ones and that teachers change their point of view and show a positive attitude after working with computer assisted exam interfaces.

Keywords: attitudes, PAU (University Entry Exam), evaluation, oral skills, methodology.

SUMARIO: Introducción. 1. Revisión de proyectos realizados en la Comunidad Valenciana. 2. Diseño e implementación de tareas orales. 3. Factores favorables y desfavorables de la prueba oral en la PAU. 4. Conclusiones. Referencias bibliográficas.

\section{INTRODUCCIÓN}

La comunicación por parte del Ministerio del nuevo Real Decreto, por el que se regulan las condiciones para el acceso a las enseñanzas universitarias oficiales de grado y los procedimientos de admisión a las universidades públicas españolas que 
entrará en vigor a partir del curso 2009/10, ha suscitado evidentemente mucha inquietud en el entorno de la enseñanza secundaria en España. Este Real Decreto contempla en su artículo 9 la incorporación de un ejercicio de lengua extranjera cuyo objetivo es valorar la comprensión oral y lectora, además de la expresión oral y escrita. Debido a que la evaluación de la comprensión y expresión oral del estudiante es un componente nuevo en el examen de lengua extranjera, los docentes de la enseñanza secundaria empiezan a replantearse cómo tendrán que adaptar su metodología docente. Pese a que el nuevo modelo de prueba entrará en vigor en el curso 2009/10, la prueba oral correspondiente al examen de lengua extranjera no se implantará hasta el curso 2011/2012. Varios proyectos en la Comunidad Valenciana han permitido la realización de estudios que demuestran que es posible introducir la evaluación de las destrezas orales en la Prueba de Acceso a la Universidad.

El grupo de investigación CAMILLE (Universidad Politécnica de Valencia) actualmente trabaja en el desarrollo de una plataforma con el objetivo de hacer viable la evaluación asistida por ordenador de los conocimientos de segundas lenguas. Esta herramienta podría ser especialmente útil y práctica a la hora de introducir la evaluación de destrezas orales. La evaluación de las mismas en el examen de lengua extranjera de la PAU obligaría a plantearse qué tipo de ejercicios podrían incluirse, y cuál sería el modo más factible para examinar tales competencias. Este trabajo forma parte del desarrollo que sigue la plataforma de exámenes PAU-ER dentro del proyecto PAULEX, la evolución natural del proyecto PLEVALEX (García Laborda 2005). Se trata de una herramienta destinada a la evaluación oral y escrita asistida por ordenador.

Entre los aspectos positivos que se destacarán a continuación, se encuentran la validez del constructo en plataformas digitales, la confianza en una evaluación a través de entrevistas semi-asistidas, la autenticidad impuesta por las tareas basadas en repertorios audiovisuales, el alto grado de interactividad conseguido en los proyectos que se han desarrollado o que están en proceso, el impacto y efecto washback en el aula, y la evidente practicidad y sencillez que supone para el alumno examinado el uso de un sistema basado en la informática lingüística.

\section{REVISIÓN DE PROYECTOS REALIZADOS EN LA COMUNIDAD VALENCIANA}

En 2004, el grupo de investigación GILFE comenzó a desarrollar el primer examen específico de idiomas con secciones orales asistido por ordenador creado en la Comunidad Valenciana. Aquel primer proyecto lo llevó a cabo una empresa privada con sede en Madrid, bajo la supervisión y las directrices del Grupo GILFE. Su desarrollo terminó en 2006 y el período de prueba se prolongó hasta mediados de 2008. El proyecto generó una veintena de artículos, algunos de los cuales todavía están en proceso de publicación. El proceso demostró la viabilidad de una prueba de idiomas asistida por ordenador dentro de la Prueba de Acceso a la Universidad 
(García Laborda y Magal Royo 2007). El modelo de examen propuesto sugería tres dimensiones comunicativas (Bachman 1990):

a. Social. Sería el tipo de comunicación oral establecida entre compañeros de universidad, amigos, etc. Incluiría una prueba de preguntas generalmente cortas.

b. Académica (comprensiva). Sería una comunicación con fines educativos, tales como descripciones o asistencia a una clase. Se dividiría en tareas con toma y comprensión de datos (como una conferencia) y en descripciones de situaciones (como un diagrama).

c. Académica (para aprendizaje). Sería aquella en la que el alumno es capaz de relacionar distintos tipos de ínput (oral y escrito) para poder expresar ese aprendizaje oralmente. Este modo se basa en la integración de distintos tipos de tareas para solucionar y reflexionar sobre un tema, demostrando la capacidad del alumno de comprender y asimilar contenidos.

Posteriormente, esta plataforma se integró en la plataforma PLEVALEX. Dicha integración y el resto de aspectos fundamentales incluidos o característicos de tal plataforma ya han sido descritos por Jesús García Laborda (2007). El proyecto se centró en la Prueba de Acceso a la Universidad (PAU), simulando las pruebas para la PAU de la Comunidad Valenciana recogidas en la web de la PAU de inglés de la Universidad Politécnica de Valencia (http://www.upv.es/ingles/).

Desde 2008 se desarrolla la plataforma PAU-ER, dentro del proyecto PAULEX Universitas, subvencionado por el Ministerio de Ciencia e Innovación (HUM200766479-C02-01), y del proyecto Selector, subvencionado por la Generalitat Valenciana (GV2007/189), para el estudio de la viabilidad de la prueba con profesores y alumnos de $2^{\circ}$ de Bachillerato.

\section{DISEÑO E IMPLEMENTACIÓN DE TAREAS ORALES}

Son numerosas las alternativas que harían posible la implantación de la evaluación de las destrezas tomadas en consideración en el presente artículo. Se plantea por tanto, en este momento de desarrollo de la herramienta PAU-ER, qué tipo de metodología de evaluación y qué tipología de ejercicios resultarían más efectivas y eficientes para testar el conocimiento oral de una lengua: la fluidez en la utilización de la gramática; el vocabulario al que se recurre y la rapidez con la que la mente elige un término u otro dependiendo de cada situación; la capacidad de descripción de una situación o imagen; la práctica conversacional a modo de entrevista o charla; etc.

El Real Decreto 1892/2008 del 14 de noviembre, mencionado al comienzo de este artículo, señala en el apartado 3 del artículo 9 que "el tercer ejercicio [de la fase general] será de lengua extranjera y tendrá como objetivo valorar la comprensión oral y lectora y la expresión oral y escrita" y que "el ejercicio presentará dos opciones diferentes entre las que el estudiante deberá elegir una." Estas son las únicas pautas generales que se conocen hasta el momento y que conducen a la reflexión sobre qué tipología de ejercicios podría resultar más clarificadora y discriminatoria del nivel de los alumnos. También plantea la cuestión de cómo podría llevarse a cabo tal 
evaluación. Las dos opciones claramente distanciadas y que posibilitarían examinar los conocimientos orales de un estudiante que acabara de finalizar los estudios de Bachillerato serían, por un lado, un tribunal presencial que valorara in situ los conocimientos del alumno $\mathrm{y}$, por otro, la evaluación mediante sistemas informáticos avanzados. Los estudios que han fundamentado y guiado tanto la creación de la plataforma PLEVALEX como la de su evolución natural, PAU-ER, avalan la posibilidad de crear una plataforma que haga viable la introducción de la evaluación de las destrezas orales en el examen de lengua extranjera de la PAU. La grabación de voz, en el caso de la producción oral, ofrecería datos reales y tangibles de la producción de cada estudiante para que un examinador los valorase y puntuase. Este también es un factor clave para que el estudiante pueda pedir una revisión o una segunda valoración de sus resultados.

Barry O'Sullivan (2008) llevó a cabo recientemente una recopilación de ejercicios que se han empleado para la evaluación de las habilidades orales en diversos ámbitos. Este completo análisis y repaso de los diferentes modos de evaluar las destrezas orales ha sido tomado como base principal para plantear algunos de los ejercicios que proponemos para la futura pero cercana implantación de la oralidad dentro de la Prueba de Acceso a la Universidad en España. Si lo que se pretende valorar es la pronunciación, uno de los modos de mayor consistencia podría ser la lectura en voz alta de un texto o de frases que se correspondan con el nivel de los estudiantes que acaban de finalizar Bachillerato. La grabación de tales repeticiones permitiría la posterior escucha, análisis y valoración de la producción de cada estudiante. Si la intención es medir la fluidez y la calidad de un idioma hablado a través de la improvisación, la descripción de una situación o imagen alternativa podría resultar el modo más eficiente.

En algunas de las reuniones mantenidas con una importante y numerosa representación de profesores de inglés de bachillerato de la provincia de Valencia, gran parte de ellos encargados de la preparación de sus estudiantes para la Prueba de Acceso a la Universidad, ha quedado patente el temor que la evaluación de los conocimientos orales suscita entre el profesorado, por diversas razones. Muchos de ellos han señalado que la grabación de la producción oral de un estudiante en el nivel de bachillerato sólo tendría sentido y sería tolerable mediante la preparación de un discurso concreto para su reproducción frente a un micro en el momento del examen. Algunos de los profesores consideran que, por el momento, sería negativo que un estudiante con ese nivel de lengua se enfrentase y quedase expuesto a la improvisación ante un micrófono.

La otra opción en el caso de la Selectividad española, la de la exposición de un estudiante frente a un tribunal, podría tener lugar a través de una entrevista oral sobre preguntas generales; o mediante la producción de un discurso preparado sobre un tema concreto que forme parte de un listado predeterminado, el cual habría sido facilitado previamente a los centros educativos de secundaria. De este modo, los profesores y los alumnos habrían tenido tiempo para la práctica y preparación del examen. La modalidad de una entrevista o conversación a través de un sistema interactivo -y no frente a personas físicas, como sería el caso de un tribunal- obligaría 
también a seleccionar una serie de temas posibles para la preparación previa por parte de los estudiantes, de tal manera que sepan de antemano qué tipo de preguntas podrían formularse mediante esa evaluación informatizada. De este modo, ya contarían con vocabulario y fluidez suficientes como para producir una respuesta rápida, que sería grabada. Podría ocurrir que la relación persona-máquina en la evaluación de destrezas comunicativas no fuera de tanta calidad como la relación persona-persona que se produciría frente a un tribunal, debido a que, mientras que en la relación examinando-examinador se utilizaría una entrevista con preguntas variables, en la evaluación frente al ordenador se emplearía una batería fijada de preguntas y respuestas que, generalmente, son invariables.

Los resultados de diversos estudios sobre las actitudes de los alumnos hacia cursos y exámenes informatizados que se están llevando a cabo en la Universidad Politécnica de Valencia están siendo prometedores. En futuros estudios sería adecuado seguir observando y profundizando en ellos, con el fin de averiguar qué tipo de situación conllevaría un menor grado de ansiedad, y explorar tanto los niveles de estrés y nerviosismo que podría provocar una prueba de acceso a la universidad asistida por ordenador, como sus posibles consecuencias. También es necesario considerar qué ventajas y desventajas representa cada sistema de evaluación, teniendo en cuenta el amplísimo volumen de estudiantes que han de ser examinados de forma simultánea. Las ventajas más destacables de una Prueba de Acceso a la Universidad informatizada son su posible realización simultánea por parte de todos los alumnos, así como la facilitación del almacenamiento y gestión de resultados para su posterior corrección. Por otro lado, se ha de tener en cuenta que la informatización de un examen de estas características puede contar con el riesgo, aunque mínimo, de un mal funcionamiento de alguno de los ordenadores individuales. Con el fin de minimizar este riesgo, sería recomendable hacer una validación automática inicial de todos los dispositivos informáticos que intervienen. Mediante este protocolo de actuación, el examinando cuyo ordenador presentara algún tipo de problema, podría cambiar de terminal antes del comienzo de la prueba.

La introducción de la evaluación de la comprensión oral mediante sistemas informáticos es a priori la parte que plantea una incorporación más sencilla, teniendo en cuenta, entre otras cosas, la cantidad de exámenes oficiales que ya la ofrecen con éxito hoy en día. Uno de los principales requisitos es la creación de espacios virtuales con capacidad suficiente como para que puedan acceder a ellos numerosos estudiantes de forma simultánea, sin que se vea por ello mermada la calidad de los contenidos. El modo más efectivo y discriminatorio podría ser la visualización de un video o la escucha de un audio con una temática también preestablecida y que haya permitido su preparación previa en el aula. Además, las preguntas de comprensión se responderían por medio de un cuestionario también informatizado, basado en preguntas cortas o tipo test. Los exámenes oficiales que ya han recurrido a la informatización, para amoldarse a los nuevos tiempos y necesidades, avalan la efectividad de este tipo de evaluación. 


\section{FACTORES FAVORABLES Y DESFAVORABLES DE LA PRUEBA ORAL EN LA PAU}

Muchos estudios señalan que la evaluación de las destrezas orales es uno de los aspectos más controvertidos y complejos dentro del campo de la enseñanza de segundas lenguas (O'Sullivan 2008). Esto en parte se debe a la dificultad de aunar los objetivos de evaluación con las tareas o instrumentos adecuados para la misma (Luoma 2004).

Ya hemos mencionado anteriormente la atención que empieza a prestarse a las destrezas orales por parte del Gobierno central, con iniciativas tales como la introducción de su evaluación en la Prueba de Acceso a la Universidad, a partir del curso académico 2011/2012. Son muchas las voces que advierten de la falta de validez global de la enseñanza de una segunda lengua si se obvian el aprendizaje y la evaluación de las destrezas orales, como está ocurriendo actualmente en los estudios preuniversitarios.

La comprensión y la producción orales son consideradas por muchos sectores como una parte fundamental, si no la principal, en una sociedad globalizada y de amplísima movilidad internacional en la que el dominio de al menos dos lenguas extranjeras, tanto a nivel oral como escrito, se divisa como un requisito fundamental en el ámbito académico y profesional (García Laborda, Fernández Álvarez y Martínez Sáez 2008). La introducción de exámenes o tests de conversación puede considerarse como una inyección de autenticidad dentro de la evaluación de conocimientos. Podría ser además un aliciente para la utilización de herramientas interactivas destinadas al entrenamiento de tales destrezas, y tendría también un altísimo impacto en la fase de preparación para determinados exámenes, tanto en alumnos como en profesores (Akiyama 2003). Esto es algo que obligaría a su práctica, fijando como objetivo la superación de tal prueba; y que fomentaría sin duda la práctica y el nivel de conocimientos orales de los estudiantes que finalizan los estudios de bachillerato en España.

Se ha hecho mención anteriormente a la oposición inicial de algunos profesores que ejercen en la Comunidad Valenciana y que han participado en reuniones sobre la Prueba de Acceso a la Universidad. La principal queja que se plantea en este tipo de reuniones es la de la imposibilidad de la práctica y preparación de los estudiantes de bachillerato en destrezas orales, debido a que en las aulas generalmente los grupos son muy numerosos. También se ha hecho referencia al inconveniente de la falta de medios informáticos en los centros educativos de carácter público. Ante estas dificultades, los profesores se resisten a creer que la evaluación de las destrezas orales pueda ser implementada en el año 2011, pues consideran que esta fecha es prematura.

Los autores quisieran agradecer al Ministerio de Educación por la financiación de este estudio a través del proyecto de investigación (MEC HUM2007-66479-C0201/FILO) y desarrollo nacional de 2007 supervisado por la Dra. Emilia V. Enríquez Carrasco. 


\section{CONCLUSIONES}

Los datos obtenidos hasta el momento a partir de las pruebas y simulacros con la plataforma PLEVALEX, la predecesora de PAU-ER, llevados a cabo con más de 35 alumnos y 25 profesores (García Laborda, López Santiago y Martínez Sáez 2009) han demostrado que los alumnos no sienten demasiada extrañeza ante la realización de exámenes de lenguas en el emulador, posiblemente debido a que son nativos informáticos. Asimismo, los profesores se muestran menos reacios a este tipo de innovaciones cuando reciben información concreta o se les plantean cursos de formación en este campo, con lo que dejan de ver el objetivo de la evaluación oral como una meta lejana e imposible. Los resultados del repaso de las propuestas que pueden ser factibles ante la implantación de la evaluación de las destrezas orales prueban la utilidad y la viabilidad del enfoque y el proceso de estudio que se viene siguiendo en el desarrollo de las plataformas que se han mencionado. El tipo de dispositivo de evaluación en el que se está trabajando actualmente puede ser muy útil para alumnos que requieran especial motivación, y para aquéllos que se sientan especialmente atraídos hacia el juego interactivo educativo. En la actualidad, continúan llevándose a cabo diversos estudios y se sigue desarrollando este sistema de evaluación, con el objetivo de conseguir un resultado final que beneficie a los alumnos en su uso, tanto en un contexto de evaluación como de preparación para la mencionada prueba en lengua extranjera.

\section{REFERENCIAS BIBLIOGRÁFICAS}

AKIYAMA, T. (2003): "Assessing speaking in Japanese junior high schools: Issues for the senior high school entrance examinations», en SHIKEN: JALT Testing \& Evaluation SIG Newsletter http://jalt.org/test/pub.htm (07/01/2009).

BACHMAN, L. (1990): Fundamental considerations in language testing, Oxford, Oxford University Press.

GARCÍA LABORDA, J. (2005): «PLEVALEX - A new platform for oral testing in Spanish», en the Eurocall Review, Issue Number 9,

http://www.eurocall-languages.org/news/newsletter/9/index. html (10/12/2008).

GARCÍA LABORDA, J. (2007): «From Fulcher to PLEVALEX: Issues in Interface design, validity and reliability in Internet based Language Testing», en Computer Assisted Language Learning EJ, 9(1): 1-9.

GARCÍA LABORDA, J. Y MAGAL ROYO, T. (2007): «Diseño y validación de la plataforma PLEVALEX como respuesta a los retos de diseño de exámenes de lenguas para fines específicos», en Ibérica, 14: 59-78.

GARCÍA LABORDA, J., FERNÁNDEZ ÁLVAREZ, M. Y MARTÍNEZ SÁEZ, A. (en prensa): «Retos actuales de la Prueba de Acceso a la Universidad en lenguas extranjeras ante una sociedad paneuropea», X Congreso Internacional de la Sociedad Española de Didáctica de la Lengua y la Literatura (SEDLL), Universidad de La Laguna, Santa Cruz de Tenerife, 3-5 diciembre 2008. 
GARCÍA LABORDA, J., LÓPEZ SANTIAGO, M. Y MARTÍNEZ SÁEZ, A. (en prensa): «Student's attitudes towards a French Computer Assisted test». Eurocall Conference, Gandía (España) 9-11 septiembre 2008.

LUOMA, S. (2004): Assessing Speaking, New York, Cambridge University Press.

O'SULLIVAN, B. (2008): «Notes on assessing speaking» Cornell University - Language Resource Center, http://rc.cornell.edu/events/past/2008-2009/papers08/osull1.pdf $(10 / 01 / 2009)$. 